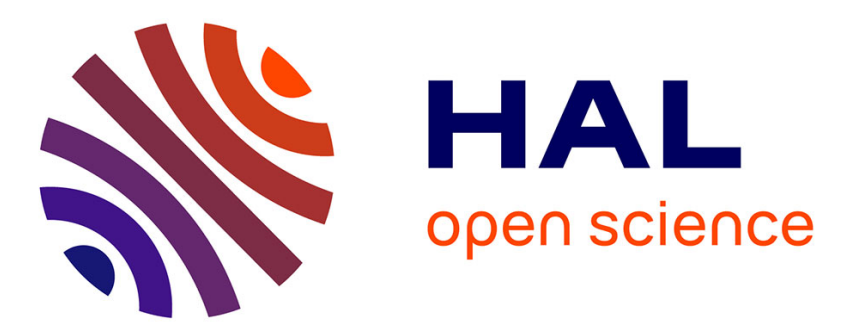

\title{
A gradient-based optical-flow cardiac motion estimation method for cine and tagged MR images
}

Liang Wang, Patrick Clarysse, Zhengjun Liu, Bin Gao, Wanyu Liu, Pierre Croisille, Philippe Delachartre

\section{- To cite this version:}

Liang Wang, Patrick Clarysse, Zhengjun Liu, Bin Gao, Wanyu Liu, et al.. A gradient-based opticalflow cardiac motion estimation method for cine and tagged MR images. Medical Image Analysis, 2019, 10.1016/j.media.2019.06.016 . hal-02168613

\section{HAL Id: hal-02168613 \\ https://hal.science/hal-02168613}

Submitted on 25 Oct 2021

HAL is a multi-disciplinary open access archive for the deposit and dissemination of scientific research documents, whether they are published or not. The documents may come from teaching and research institutions in France or abroad, or from public or private research centers.
L'archive ouverte pluridisciplinaire HAL, est destinée au dépôt et à la diffusion de documents scientifiques de niveau recherche, publiés ou non, émanant des établissements d'enseignement et de recherche français ou étrangers, des laboratoires publics ou privés.

\section{()ㅜ(1)}

Distributed under a Creative Commons Attribution - NonCommerciall 4.0 International 


\title{
A gradient-based optical-flow cardiac motion estimation method for cine and tagged MR images
}

\author{
Liang Wang ${ }^{\mathrm{a}}$, Patrick Clarysse ${ }^{\mathrm{a}}$, Zhengjun $\mathrm{Liu}^{\mathrm{c}}$, Bin Gao ${ }^{\mathrm{c}, \mathrm{d}}$, Wanyu Liuc ${ }^{\mathrm{c}}$, Pierre Croisille ${ }^{\mathrm{a}, \mathrm{b}}$, Philippe Delachartre ${ }^{\mathrm{a}}$ \\ ${ }^{a}$ Univ Lyon, INSA-Lyon, Université Lyon 1, UJM-Saint Etienne, CNRS, Inserm, CREATIS UMR 5220, U1206, F-69621, LYON, France \\ ${ }^{b}$ Department of Radiology, University Hospital of Saint-Etienne, Université Jean-Monnet, Saint-Etienne, France \\ ${ }^{c}$ Metislab, LIA CNRS, Harbin Institute of Technology, Harbin 150001, People's Republic of China \\ ${ }^{d}$ College of data science and technology, Heilongjiang University, Harbin 150080, People's Republic of China
}

\begin{abstract}
A new method is proposed to quantify the myocardial motion from both $2 D$ C(ine)-MRI and T(agged)-MRI sequences. The tag pattern offers natural landmarks within the image that makes it possible to accurately quantify the motion within the myocardial wall. Therefore, several methods have been proposed for T-MRI. However, the lack of salient features within the cardiac wall in C-MRI hampers local motion estimation. Our method aims to ensure the local intensity and shape features invariance during motion through the iterative minimization of a cost function via a random walk scheme. The proposed approach is evaluated on realistic simulated C-MRI and T-MRI sequences. The results show more than 53\% improvements on displacement estimation, and more than $24 \%$ on strain estimation for both C-MRI and T-MRI sequences, as compared to state-of-the-art cardiac motion estimators. Preliminary experiments on clinical data have shown a good ability of the proposed method to detect abnormal motion patterns related to pathology. If those results are confirmed on large databases, this would open up the possibility for more accurate diagnosis of cardiac function from standard C-MRI examinations and also the retrospective study of prior studies.
\end{abstract}

Keywords: myocardial motion estimation, gradient-based optical flow, local shape, C-MRI and T-MRI

\section{Introduction}

Assessment of cardiac function is of a primary importance for care of patients with heart disease. In addition to global parameters, such as ejection fraction, regional quantification of motion is helpful to locally characterize the myocardium in ischemic heart diseases. Cardiac magnetic resonance imaging (MRI), in particular cine-MRI (C-MRI), is an advantageous imaging modality to assess cardiac function. Tagged-MRI (TMRI) is considered as the reference for regional cardiac motion quantification (Wang and Amini, 2012), although not used in clinical practice.

Numerous approaches have been proposed for either C-MRI and T-MRI sequences processing (Miao et al., 2016; Dosenbach et al., 2017; Küstner et al., 2017; Parages et al., 2017; Tolouee et al., 2018). The harmonic phase image method was introduced by Osman and Prince in early 2000 and became the standard method for T-MRI analysis (Osman et al., 1999). Since then, improvements to the original method have been proposed, e.g., the local sine wave modeling method (SinMod) (Arts et al., 2010), the monogenic-signal-based methods (Alessandrini et al., 2013; Gao et al., 2016) and the analytic signal phasebased method (Wang et al., 2015). The optical flow-based principle has been adapted to T-MRI processing to account for intensity variations (Prince and McVeigh, 1992), or within 3D multi-resolution (Xu et al., 2010) approaches. Because of the lack of structural information, quantification of the motion in C-MRI requires complementary constraints, e.g., physical con- straints such as incompressibility (Bistoquet et al., 2007), geometrical/intensity constraints that can be integrated into an attribute vector to control registration in image sequences (Delhay et al., 2007; Sundar et al., 2009). There is clear interest for motion estimation methods from T-MRI and C-MRI: T-MRI is the gold standard for motion quantification and C-MRI is acquired routinely in the clinic. Indeed, motion estimation is usually independently addressed relative to a specific MR acquisition: cine, tagged, phase contrast, displacement encoding with stimulated echoes (DENSE), or strain encoding (SENC) (Wang and Amini, 2012).

Moreover, gradient-based motion estimation plays an important role in registration algorithms, which have been considered independently for T-MRI or C-MRI sequence motion estimation (Keller and Averbuch, 2004). Hence, gradient-based optical flow strategy could be a possible framework for developing a C-MRI and T-MRI sequence compatible motion estimation method. The gradient constancy assumption has been proposed to handle image illumination changes as a complement to the traditional brightness constancy assumption (Brox et al., 2004; Zimmer et al., 2011). A very large scale integration (VLSI) architecture of the multi-channel gradient model (McGM) proposed by Johnston et al. has also been implemented (Botella et al., 2012). Boonsieng et al. adopted the orientation of the gradient instead of gradient intensity for a robust optical flow estimation, within a method called the gradient orientation-based gradient method (GOGM) (Boonsieng et al., 2014). Revaud et al. also introduced the gradient-constancy assumption in a 
variational energy minimization function for optical flow estimation integrating descriptor matching (Revaud et al., 2015). In (Bristow and Lucey, 2016), Bristow and Lucey discussed the gradient-based method by regression for image alignment, illustrating that image alignment can be used as an optical flow estimation method.

In this paper, we propose a method that can equally process both C-MRI and T-MRI sequences. Firstly, a geometrical intensity distribution model is defined by local mean intensity and gradient. Then, invariance of the local model parameters on both intensity and gradient features is imposed through a system of four optical flow equations. An overall cost function is defined based on the invariance equations, the minimization of which results in the optimized displacement between two consecutive images. The proposed method is evaluated on both simulated and clinical, C-MRI and T-MRI sequences. A method is proposed to create a T-MRI sequence from a simulated C-MRI sequence, animated by the same motion. The motion estimation results obtained with the proposed method outperform those of other state-of-the-art methods with the simulated data. Furthermore, the proposed method is applied to the clinical data from a healthy volunteer and an acute myocardial infarction patient for myocardial deformation analysis. In this preliminary experiments, the proposed method's performance compares favorably to state-of-the-art methods for both C-MRI and T-MRI sequences.

The paper is organized as follows. In section 2, the proposed method based on optical flow equations is detailed. In section 3, the synthetic method for T-MRI sequences is introduced. The detailed implementation is described in section 4 and the results obtained on synthetic and clinical data are given and discussed.

\section{Motion estimation method}

The proposed method estimates the motion field between two successive images. It is based on the traditional brightness constancy combined with a local shape constraint. A random walk optimization is adopted to estimate the optimal displacement field. The accumulated motion field over the temporal image sequence is calculated as well for motion analysis and strains computation.

\subsection{Pixel intensity and local shape constraints}

The traditional optical flow method is based on the assumption of intensity invariance of material points during motion. For two consecutive images, $I_{1}$ and $I_{2}$, this assumption is translated by the relation:

$$
I_{1}(\mathbf{x}+\mathbf{v}, t+d t)=I_{2}(\mathbf{x}, t)
$$

where $\mathbf{x}=(x, y)$ and $\mathbf{v}=(u, v)$. The $(x, y)$ is the spatial coordinates and $(u, v)$ represent the horizontal and vertical velocity, respectively. Under the small motion assumption, the brightness constancy constraint in Eq. (1) can be expressed by the optical flow constraint equation:

$$
\nabla I \cdot \mathbf{v}+I_{t}=0
$$

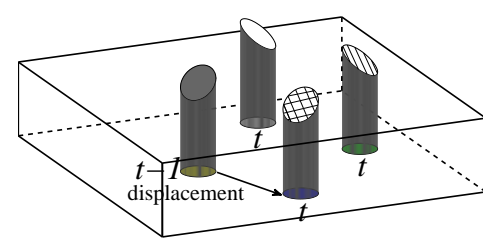

(a)

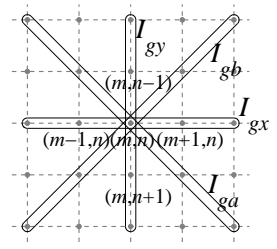

(b)
Figure 1: (a) The local shape model. For the gray cylinder at time $t-1$, there are three possible displacements at time $t$ the intensity invariance constraint only (white, line- and grid-textured cylinders) and a single solution considering both the intensity and local shape information. (b) Diagram for the discrete approximation of the intensity gradients $\left(I_{g x}, I_{g y}, I_{g a}, I_{g b}\right)$ in four directions $(x, y, a, b)$.

where $\nabla I$ is the gradient of the intensity and $I_{t}$ is the intensity temporal derivative. However, Eq. (2) is not sufficient to solve for the two motion components. As a result, the local shape of each pixel is considered as a complementary intrinsic property. As shown in Fig. 1(a), the local shape is represented by the approximated intensity plane given by the local spatial intensity gradient $\left(I_{g x}\right.$ and $I_{g y}$ in Fig. 1(b), which defines the slope of the local intensity patch). The four cylinders have the same central intensity level but present different local shapes. For the marked in gray at time $t-1$, there are three possible positions at time $t$ based on Eq. (1) only, which are marked in white or with lines and grids. However, the local slopes for these three elliptic surface patches are not the same. Hence, the grid-textured in Fig. 1(a) is the only correct result when considering both the local intensity and the local shape information.

Both the intensity (Eq. (1)) and local shape constraints should be satisfied simultaneously, leading to the two additional equations:

$$
I_{1, g x}(\mathbf{x}+\mathbf{v})=I_{2, g x}(\mathbf{x}), \quad I_{1, g y}(\mathbf{x}+\mathbf{v})=I_{2, g y}(\mathbf{x}),
$$

where $I_{g x}, I_{g y}$ are the gradients of $I$ in $x$ and $y$ directions, respectively. In addition to the horizontal and vertical gradients, invariance of two diagonal gradients, $I_{g a}$ and $I_{g b}$, is required as well:

$$
I_{1, g a}(\mathbf{x}+\mathbf{v})=I_{2, g a}(\mathbf{x}), \quad I_{1, g b}(\mathbf{x}+\mathbf{v})=I_{2, g b}(\mathbf{x}) .
$$

The four gradients are computed together with a five-point numerical approximation (Fig. 1(b)). They represent the local patch slope at location $(m, n)$ :

$$
\begin{aligned}
I_{g x}[m, n]= & \frac{1}{12}(I[m-2, n]-8 I[m-1, n] \\
& +8 I[m+1, n]-I[m+2, n]), \\
I_{g y}[m, n]= & \frac{1}{12}(I[m, n-2]-8 I[m, n-1] \\
& +8 I[m, n+1]-I[m, n+2]), \\
I_{g a}[m, n]= & \frac{1}{12 \sqrt{2}}(I[m-2, n-2]-8 I[m-1, n-1] \\
& +8 I[m+1, n+1]-I[m+2, n+2]), \\
I_{g b}[m, n]= & \frac{1}{12 \sqrt{2}}(I[m+2, n-2]-8 I[m+1, n-1] \\
& +8 I[m-1, n+1]-I[m-2, n+2]),
\end{aligned}
$$

In addition, local shape gradients are smoothed through to the 
convolution with a $2 D$ Gaussian filter.

$$
\begin{aligned}
I_{x}[x, y] & =I_{g x}[x, y] * * g[x, y], \\
I_{y}[x, y] & =I_{g y}[x, y] * * g[x, y], \\
I_{a}[x, y] & =I_{g a}[x, y] * * g[x, y], \\
I_{b}[x, y] & =I_{g b}[x, y] * * g[x, y],
\end{aligned}
$$

where $* *$ denotes the discrete $2 D$ convolution and $g[x, y]$ is the discrete Gaussian kernel. These smoothed local shapes are used in the following processing.

\subsection{Optimized displacement estimation by a random walk ap- proach}

Random walk scheme refers to a special category of Markov chains and can be used to scan multi-dimensional space. It has been notably experimented for motion prediction (Harris, 1989; Kawamoto, 2007). Taking a pixel of an image at the position $\mathbf{x}_{t-1}$ at time $t-1$ as a particle, the random walk model simulates the probability of the pixel displacement at time $t$ to its neighbor position $\mathbf{x}_{t}$ :

$$
\mathbf{x}_{t}=\mathbf{x}_{t-1}+\mathbf{p}_{t},
$$

where $\mathbf{p}_{t}$ is $2 D$ white Gaussian noise with a normal probability distribution. Therefore, the displacement field $\mathbf{v}=(u, v)$ can be estimated with a random walk optimization of the system in Eqs. (5) and (6).

The displacement magnitude is restricted to $\left[0, r_{\max }\right]$, where $r_{\text {max }}$ is defined as the maximum magnitude of possible random displacement. A random displacement data set of $(u, v)$ is proposed, with the constraint $(u, v) \in\left[-r_{\text {max }}, r_{\text {max }}\right]$, which is obtained from the random walk model and guided by Eqs. (1), (3) and (4).

In an image $I_{1}(\mathbf{x})$, a random displacement $\mathbf{v}=(u, v)$ is applied to each pixel $\mathbf{x}=(x, y)$ of $I_{1}(\mathbf{x})$, which generates an image $\widehat{I_{2}}(\mathbf{x})$ :

$$
\widehat{I_{2}}(\mathbf{x})=I_{1}(\mathbf{x}+\mathbf{v}) .
$$

$\widehat{I_{2}}(\mathbf{x})$ defines interpolation of four points of $I_{1}$, which are the point $\mathbf{x}+\mathbf{v}$ and its four adjacent points. The motion field is iteratively updated to minimize the following cost function $E(\mathbf{x}, \mathbf{v})$ :

$$
\begin{aligned}
& E(\mathbf{x}, \mathbf{v})=\sum_{\forall k}\left|\widehat{I_{2, k}}(\mathbf{x})-I_{2, k}(\mathbf{x})\right| \\
& =\sum_{\forall k}\left|I_{1, k}(\mathbf{x}+\mathbf{v})-I_{2, k}(\mathbf{x})\right|, k=i, x, y, a, b
\end{aligned}
$$

where " $i$ " is the image intensity distribution, and $x, y, a$ and $b$ indexes the four gradient directions.

A series of random displacement propositions is applied to each pixel. For a given pixel $\mathbf{x}$ and iteration iter, a random displacement $\mathbf{v}=\left(u^{(i t e r)}, v^{(i t e r)}\right)$ is proposed first. The parameter iter is the iteration index of the random displacement proposal. If the pixel's cost function $E^{(i t e r)}(\mathbf{x}, \mathbf{v})$ obtained from Eq. (9) is smaller than the reference cost function $E^{(r e f)}(\mathbf{x}, \mathbf{v})$, then the expected displacement $(u, v)$ of the given pixel $\mathbf{x}$ is updated with $\left(u^{(i t e r)}, v^{(i t e r)}\right)$, and the reference cost function $E^{(r e f)}(\mathbf{x}, \mathbf{v})$ is updated with $E^{(i t e r)}(\mathbf{x}, \mathbf{v})$. Otherwise, no updating is applied at this iteration. The initial value of reference cost function $E_{\text {init }}^{(r e f)}(\mathbf{x}, \mathbf{v})$ is given a large number. This process will stop when the iteration index reaches the maximum iteration number iter $_{\text {max }}$, an optimized $\mathbf{v}=(u, v)$ is obtained for the pixel $\mathbf{x}$. An optimal dense displacement between two successive frames is obtained once all the image pixels have been processed.

Moreover, we assume that the motion within a local neighborhood is continuous, within a small block around a pixel. Therefore, a smoothed estimation of the energy in Eq. (9) is obtained by:

$$
\bar{E}\left(\mathbf{x}_{m_{0}, n_{0}}, \mathbf{v}\right)=\frac{1}{9} \sum_{m, n \in \Omega} w(\alpha)_{m, n} E\left(\mathbf{x}_{m, n}, \mathbf{v}\right)
$$

with

$$
\omega(\alpha)=\frac{4}{1+\alpha}\left[\begin{array}{ccc}
\frac{\alpha}{4} & \frac{1-\alpha}{4} & \frac{\alpha}{4} \\
\frac{1-\alpha}{4} & 1 & \frac{1-\alpha}{4} \\
\frac{\alpha}{4} & \frac{1-\alpha}{4} & \frac{\alpha}{4}
\end{array}\right]
$$

where $\Omega$ is the nine-pixel neighborhood with its center pixel at $\left(m_{0}, n_{0}\right)$. Matrix $\omega$ represents a Laplacian filter with parameter $\alpha$ in $[0,1] . E\left(\mathbf{x}_{m, n}, \mathbf{v}\right)$ is the cost function $E(\mathbf{x}, \mathbf{v})$ at pixel $(m, n)$. When a given $\mathbf{v}$ leads to a minimum of $\bar{E}$, this $\mathbf{v}$ is considered the estimated displacement of the $3 \times 3$ region of interest (ROI). As a result, a dense motion field $\mathbf{v}_{t}=\left(u_{t}, v_{t}\right)(t=1,2, \ldots)$ between two successive frames, $I_{t-1}$ and $I_{t}$, is obtained.

The process is repeated for all the successive image pairs of the sequence. Then the Lagrangian motion field is calculated using bilinear interpolation based on the successive frames' motion field. Let $\boldsymbol{L}_{t}=\left(L_{u t}, L_{v t}\right)$ represents the Lagrangian motion field between the first frame $I_{0}$ and the frame $I_{t}$ at time $t$. Furthermore, a "forward and backward" strategy is proposed in order to limit the error accumulation as much as possible when calculating the Lagrangian motion field. A forward-backward ending time frame index (FBE) is introduced as the time index of the frame, which leads the smallest error during the Lagrangian motion field calculation. Figure 2(a) illustrates the $\boldsymbol{L}_{t}$ calculation in the forward direction (black arrow) and backward direction (red arrow), respectively. The calculation of $\boldsymbol{L}_{t}$ with $t \leq \mathrm{FBE}$ used forward calculation and those with $t>$ FBE used backward calculation. In addition, the forward and backward calculations start from the same frame time index, which makes sure that no frame delay error is involved.

The following pseudo-code illustrates the steps of the proposed method.

Input: A frame set $I_{t}(t=0,1,2, \ldots, T)$

$\alpha$ : parameter of window weighting function $\omega$.

$r_{\text {max }}$ : magnitude maximum of possible displacement. $E_{\text {init }}^{(r e f)}(\mathbf{x}, \mathbf{v})$ : initial error for $E^{(r e f)}(\mathbf{x}, \mathbf{v})$.

iter $_{\text {max }}$ : maximum iteration number for random displacement proposition.

Step 1: Initialize a random displacement $\left(\mathbf{v}=u_{t}, v_{t}\right)$ and the cost function $\bar{E}$ in Eq. (10).

Step 2: Calculate the gradients $I_{x}, I_{y}, I_{a}$ and $I_{b}$ using Eq. (5) and Eq. (6). Calculate $\widehat{I_{2, k}}(\mathbf{x})(k=i, x, y, a, b)$ based on Eq. (8) using an interpolation with random displacement $\mathbf{v}$.

Step 3: Pixel-by-pixel process.

for iter=1:iter $r_{\text {max }}$

Propose a random displacement $\mathbf{v}=\left(u_{t}^{(i t e r)}, v_{t}^{(i t e r)}\right)$ smaller than $r_{\text {max }}$ for each pixel, to compute the cost function 


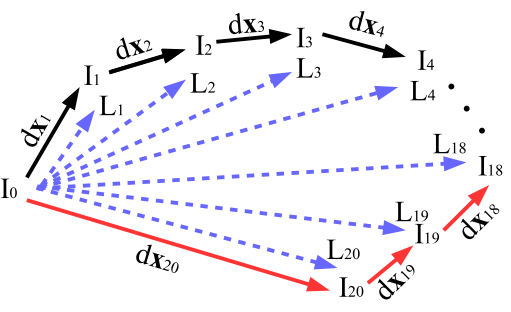

(a)

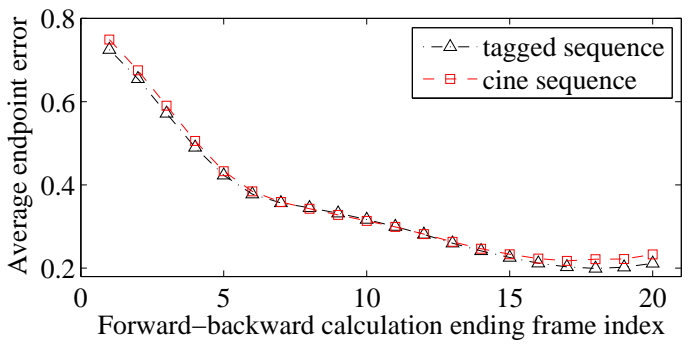

(b)

Figure 2: (a) The forward-backward scheme reduces the displacement error when computing the Lagrangian displacement field with the FBE time frame index $=18$. (b) Average EPE of the proposed method at each FBE frame from 1 to 20 with the forward and backward motion estimation scheme. $I_{18}$ and $I_{17}$ were the optimized FBE frames of the proposed method for simulated C-MRI and T-MRI sequences, respectively.

$$
\begin{aligned}
& \overline{E^{(i t e r)}}(\mathbf{x}, \mathbf{v}) \text { by Eq. (10) at each pixel. } \\
& \text { If } \overline{E^{(i t e r)}}(\mathbf{x}, \mathbf{v})<E^{(r e f)}(\mathbf{x}, \mathbf{v}) \text { : } \\
& E^{(r e f)}(\mathbf{x}, \mathbf{v}) \Leftarrow \overline{E^{(i t e r)}}(\mathbf{x}, \mathbf{v}) \\
& \mathbf{v}=\left(u_{t}, v_{t}\right) \Leftarrow \mathbf{v}=\left(u_{t}^{(i t e r)}, v_{t}^{(i t e r)}\right)
\end{aligned}
$$

endfor

Step 4: Calculate the Lagrangian displacement field $\left(L_{u t}, L_{v t}\right)$ using forward and backward motion accumulation.

Output: $\left(L_{u t}, L_{v t}\right)$ : Lagrangian displacement between $I_{0}$ and $I_{t}$ $(t=0,1,2, \ldots, T)$.

\section{Synthetic C-MRI and T-MRI sequences}

Motion estimation accuracy with the proposed approach is quantitatively evaluated from the simulation of both $2 D$ short axis C-MRI and T-MRI sequences. A C-MRI (Fig. 3(a)) is progressively deformed according to a combination of contractiondilation and rotation motion components that realistically mimic the changes in the left ventricle myocardium within a short axis slice during the cardiac cycle (Clarysse et al., 2011). T-MRI sequences are built from C-MRI sequences. They therefore contain the same ground truth motion field as the C-MRI sequence. For T-MRI sequence generation, the tag pattern is produced in the first frame by the following modulation function:

$$
\begin{array}{r}
f(x)= \begin{cases}\alpha \cos (x), & \text { if } \quad \cos (x)<T_{h}, \\
1, & \text { if } \quad \cos (x) \geq T_{h},\end{cases} \\
f_{m}(x, y)=f\left(k_{x} x+k_{y} y\right) f\left(k_{y} x-k_{x} y\right),
\end{array}
$$

where $f(x)$ is a truncated cosine function with a threshold $T_{h}$ and is regarded as a basic function for constructing the $2 D$

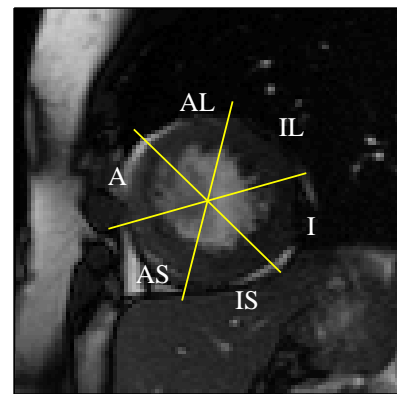

(a)

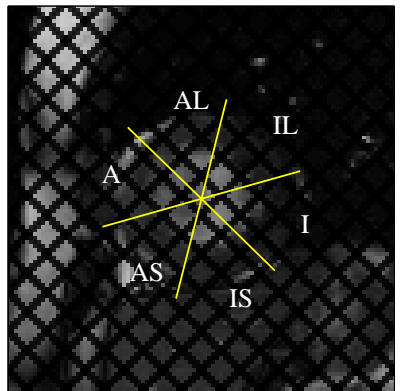

(b)
Figure 3: (a) First frame of the $2 D$ short axis C-MRI sequence. (b) First frame of the T-MRI image generated from the C-MRI sequence. The myocardium is divided into six segments. A: anterior; AS: anteroseptal; IS: inferoseptal; I: inferior; IL: inferolateral; AL: anterolateral.

modulated data. $f_{m}(x, y)$ is the $2 D$ modulation function with $k_{x}$ and $k_{y}$ the spatial frequency for the horizontal and vertical directions, respectively. In order to evaluate the robustness of the proposed method to the tag fading effect, a tag weight $\alpha$ is defined in Eq. (12). The tag fading effect has been incorporated following an experimental law as described in (Tecelão et al., 2006), the simulated tags fade exponentially up to $80 \%$ on the last frame of the sequence compared to the first frame. In our simulations, $\alpha$ does not change as a function of the pixel position in the image as it is often the case in the literature (see for instance (Zhou et al., 2018)). A preliminary study has shown that the variation of intensity across the image is not significant in our clinical cardiac T-MRI database.

The first frame of a T-MRI sequence is represented in Fig. 3(b), which is generated from Fig. 3(a) by the modulation function $f_{m}$. The successive frames of the T-MRI sequence are obtained using the wrapping operation with the ground truth displacements of the C-MRI sequence. As a result, this T-MRI sequence has the same displacement as the C-MRI sequence. The threshold $T_{h}$ was fixed to -0.6 from the experiments. The parameters $k_{x}$ and $k_{y}$ in Eq. (13) were both set to $\frac{\pi}{5}$ based on experience. The position variables $x$ and $y$ are constrained to the range [0,1]. As in (Feng et al., 2009), white Gaussian noise has been added to each frame of both C-MRI and T-MRI sequences. The signal-to-noise ratio (SNR) of each frame is set to $20 \mathrm{~dB}$.

\section{Results and discussion}

The performance of the proposed method was evaluated on synthetic and clinical as well as C-MRI and T-MRI sequences. Moreover, the proposed method was compared to a selection of state-of-the-art motion algorithms.

\subsection{Myocardium center and region mask determination}

The evaluation focuses on the left ventricle (LV) myocardium, therefore, a mask of the myocardium was needed. In the synthetic C-MRI and T-MRI sequences, the myocardium mask and center were inputs of the simulation and therefore 
known. For the clinical cases, both C-MRI and T-MRI sequences were acquired on the same patient at the same slice level. The process of determining the myocardium center and region mask is described in the following steps:

- Step 1: The myocardial region is defined by manually drawn the endocardiac and epicardiac borders in a clinical C-MRI at end-systole (see an example in Fig. 10(a)). A mask is defined to show this region.

- Step 2: The LV myocardial center is computed as the mask's center of mass.

- Step 3: The mask from the C-MRI sequence is positioned in the first frame of the corresponding T-MRI sequence based on an user-defined cavity center. A scaling factor is accounted for if clinical C-MRI and T-MRI sequences present different image resolutions. An example of manually defined masks on clinical T-MRI sequences is presented in Figs.13(a) and 18(a).

\subsection{Simulation results}

Both C-MRI and T-MRI sequences were processed to evaluate the algorithm's performance. The motion fields of the simulated image sequences served as the reference displacement for error analysis.

\subsubsection{Simulated sequences}

The simulated sequences were named CINE and CINE_NOISE for T-MRI sequences, and TAG and TAG_FADING_NOISE for C-MRI sequences. The parameters of the simulated sequences were: image size $160 \times 160$ pixels with pixel resolution $1 \times 1 \mathrm{~mm}$, contraction/expansion ratio $30 \%$, rotation 20 degrees, frame rate 20 frames per second. The total number of frames is equal to 21 , in which the first seven frames are part of the systolic phase and the next 14 frames are part of the diastolic phase of the cardiac cycle.

\subsubsection{Methods used for comparison and associated parame- ters}

The well-known method of Lucas and Kanade (LK) (Lucas and Kanade, 1981), the more recent method developed by Sun et al. (Sun) (Sun et al., 2010), and a state-of-the-art method of Gao et al. (Gao) (Gao et al., 2016) that led to more accurate performance than LK and Sun in cardiac motion estimation in C-MRI were applied to evaluate motion estimation results from both C-MRI and T-MRI sequences. For the T-MRI sequence, two more methods were considered: (1) Alessandrini's monogenic-signal-based method (MA) (Alessandrini et al., 2013), which was shown to outperform the SinMod method (Arts et al., 2010), and another monogenic-signal-based method (Zang et al., 2007) (Code courtesy of the authors); (2) and Wang's analytical-phase-based method (Wang) (Wang et al., 2015) proposed recently, which was shown to provide better motion estimation in T-MRI sequences. To estimate the Lagrangian displacement, which cumulates the motion field from successive frames, a frame index (FBE) was determined for each method to reduce the accumulation error in the computation.

\subsubsection{Evaluation}

The estimation error was evaluated with the endpoint error (EPE) metrics for each frame (Fleet and Jepson, 1990; Alessandrini et al., 2013):

$$
\mathrm{EPE}=\sqrt{\left(u_{e}-u_{c}\right)^{2}+\left(v_{e}-v_{c}\right)^{2}}
$$

where $\left(u_{e}, v_{e}\right)$ is the estimated displacement and $\left(u_{c}, v_{c}\right)$ is the ground truth at one frame. The statistics of the EPE over the myocardial mask at each time point characterize the method's accuracy.

Furthermore, strains can be computed from Lagrangian displacement fields, which are among the critical indicators for cardiac function assessment (Qian et al., 2011). The GreenLagrange strain tensor (Oubel et al., 2012) was considered. It is expressed as:

$$
\left|\begin{array}{ll}
E_{x x} & E_{x y} \\
E_{y x} & E_{y y}
\end{array}\right|=\frac{1}{2}\left(\nabla \mathbf{v}+\nabla \mathbf{v}^{t}+\nabla \mathbf{v}^{t} \nabla \mathbf{v}\right),
$$

where $\mathbf{v}=(u, v)$ is the displacement. For each pixel $(x, y)$, the maximum $E_{\text {max }}(x, y)$ and minimum $E_{\text {min }}(x, y)$ strains were computed respectively from the maximal and minimal eigenvalues of the Green-Lagrange strain tensor. $E_{\max }(x, y)$ is therefore defined as:

$$
E_{\max }(x, y)=\max \left(\lambda_{1}(x, y), \lambda_{2}(x, y)\right) .
$$

\subsubsection{Methods' parameters}

The optimized parameters in the proposed optical flow scheme and tested methods were determined from the experiments on the simulated image sequences: maximum possible displacement between two successive frames $v_{\max }=2$ pixels, window weighting function factor $\alpha=0.01$; the interpolation method used in Eq. (8) was bilinear interpolation, and the maximum iteration number iter $_{\max }=4000$. A $5 \times 5$ Gaussian smooth kernel was used in Eq. (6) with sigma=0.9. The initial value of reference cost function $E_{\text {init }}^{(r e f)}(\mathbf{x})$ was set to $10^{6}$. With Sun's method, we used the recommended parameters in (Sun et al., 2010): $5 \times 5$-pixels sobel edge detector and a neighborhood of $15 \times 15$ pixels. With Gao's method, the wavelength is 5 pixels, and the order of the highpass Butterworth filter is 3 . The pyramid factor is 0.3 with the neighborhood size of $5 \times 5$ pixels. With the LK method, the window size was $11 \times 11$ pixels. With Wang's method, the grid size of the bilinear model was $4 \times 4$ pixels, the block size of $10 \times 10$ pixels, and the maximum iteration number was set to 2 . With the MA method, the initial wavelength and refinement step number were $\lambda_{0}=4$ and $N_{p}=5$, respectively.

For the Lagrangian displacement calculation from the simulated sequences, the optimized FBE frames were evaluated in terms of minimum EPE (Eq. (14)). The FBE frame were $I_{17}$ and $I_{18}$ for C-MRI and T-MRI sequences for the proposed method, respectively (see Fig. 2(b)); Sun's method uses FBE frame $I_{16}$ and $I_{17}$ for C-MRI and T-MRI sequences, respectively; the FBE frame was $I_{14}$ for Gao's method; $I_{9}$ for the LK method, $I_{19}$ for Wang's method, $I_{18}$ for the MA method for both C-MRI and TMRI sequences. For the strain tensor calculation, a $11 \times 11$ 
Gaussian smooth filter (with sigma=1.5) was applied on the partial derivative of the Lagrangian displacement of $1 \mathrm{~mm}$ per pixel for noise reduction.

\subsubsection{C-MRI sequence results}

The box-and-whisker plot of Lagrangian EPE for the "CINE" sequence between the estimated displacements and the ground truth is shown for the four methods in Fig. 4(a).

The length of the box is the interquartile range (IQR), which represents the range of the middle $50 \%$ of the EPE data. The average Lagrangian EPE values over the whole sequence were $0.236,0.453,0.362$, and 0.917 pixels for the proposed, Sun's, Gao's, and LK methods, respectively. As a result, the Sun's, Gao's, and LK EPE errors were 92\%, 53\%, and 289\% higher than the error of the proposed method. Moreover, the dispersion of EPE error was greatly reduced with the proposed approach (shorter box extent). Figure 5 compares the estimated maximum strain eigenvalue $E_{\text {max }}$ maps obtained with the proposed and the selected methods to the ground truth at end-systole (i.e., at maximum myocardial contraction, Fig. 5(a)).

In this sequence, the induced pathological region (green region) covers the AS sector. Figure 6 presents the statistical comparison for the end-systolic principal $\left(E_{\max }\right)$ and anatomical $\left(E_{r r}\right)$ strains. For $E_{\text {max }}$ (Fig. 6(a)), the proposed method and Gao's method showed the IQR that is the most similar to the ground truth in segments A, IL, IS, and AS; the IQR of the LK method is more spread out than the others. Overall, estimates with our approach were the closest to the ground truth with a slight under-estimation. The same conclusion can be drawn for the radial strain $E_{r r}$ in Fig. 6(b). This is not surprising since $E_{r r}$ should follow $E_{\max }$, at least in healthy regions.

Table 1 shows the principal and anatomical strain error results of the compared methods over the whole myocardium for the C-MRI sequence. Comparing the average error of the total four types of strains, the Sun's, Gao's, and LK total strain errors were, respectively, $143 \%, 24 \%$, and $213 \%$ higher than the error of the proposed method. For the C-MRI sequence with noise, Table 2 shows the principal and anatomical strain error results of the compared methods over the whole myocardium. The average error over the four strain measurements obtained by the Sun's, Gao's, and LK methods were, respectively, 16\%, 125\%, and $17 \%$ higher than the proposed method.

\begin{tabular}{lcccc}
\hline & $E_{\max }$ & $E_{r r}$ & $E_{\min }$ & $E_{c c}$ \\
\hline Proposed & $\mathbf{0 . 0 5 5} \pm \mathbf{0 . 0 4 6}$ & $0.060 \pm 0.040$ & $\mathbf{0 . 0 3 5} \pm \mathbf{0 . 0 3 0}$ & $\mathbf{0 . 0 3 8} \pm \mathbf{0 . 0 3 8}$ \\
Sun & $0.122 \pm 0.096$ & $0.165 \pm 0.107$ & $0.105 \pm 0.071$ & $0.066 \pm 0.062$ \\
Gao & $0.063 \pm 0.051$ & $\mathbf{0 . 0 5 6} \pm \mathbf{0 . 0 4 0}$ & $0.060 \pm 0.052$ & $0.055 \pm 0.046$ \\
LK & $0.206 \pm 0.189$ & $0.163 \pm 0.116$ & $0.094 \pm 0.084$ & $0.125 \pm 0.114$ \\
\hline
\end{tabular}

Table 1: Average error $(\mu \pm \sigma)$ of principal $\left(E_{\max }\right.$ and $\left.E_{\text {min }}\right)$ and anatomical $\left(E_{r r}\right.$ and $\left.E_{c c}\right)$ strains at end-systole over the whole myocardium from the CINE sequence.

\subsubsection{T-MRI sequence results}

Figure 4(b) compares the Lagrangian EPE of the five methods obtained on the simulated T-MRI sequence. The average Lagrangian EPE values over the whole sequence were

\begin{tabular}{lcccc}
\hline & $E_{\max }$ & $E_{r r}$ & $E_{\min }$ & $E_{c c}$ \\
\hline Proposed & $\mathbf{0 . 1 4 7} \pm \mathbf{0 . 1 2 7}$ & $\mathbf{0 . 1 3 0} \pm \mathbf{0 . 1 0 7}$ & $0.103 \pm 0.075$ & $0.118 \pm 0.101$ \\
Sun & $0.185 \pm 0.158$ & $0.197 \pm 0.136$ & $0.099 \pm 0.076$ & $\mathbf{0 . 0 9 6} \pm \mathbf{0 . 0 8 7}$ \\
Gao & $0.448 \pm 0.668$ & $0.348 \pm 0.474$ & $0.149 \pm 0.109$ & $0.174 \pm 0.241$ \\
LK & $0.209 \pm 0.168$ & $0.017 \pm 0.116$ & $\mathbf{0 . 0 9 2} \pm \mathbf{0 . 0 7 0}$ & $0.114 \pm 0.113$ \\
\hline
\end{tabular}

Table 2: Average error $(\mu \pm \sigma)$ of principal $\left(E_{\max }\right.$ and $\left.E_{\text {min }}\right)$ and anatomical $\left(E_{r r}\right.$ and $\left.E_{c c}\right)$ strains at end-systole over the whole myocardium from the CINE_NOISE sequence.

$0.214,0.430,0.409,0.587,0.231$, and 0.468 pixels for the proposed, Sun's, Gao's, LK, Wang's and MA methods, respectively. The proposed method exhibits similar behavior to the C-MRI sequence as compared to the Sun's and LK methods. Wang's EPE was only $8 \%$ greater than the EPE of the proposed method. On the other hand, the EPEs of the Sun's, Gao's, LK, and MA methods were, respectively, $101 \%, 91 \%, 174 \%$, and $119 \%$ greater than the value obtained with the proposed method. Similar to the cine case, we obtained good performance with the proposed method compared to the other general-purpose methods.

Figure 7 presents the statistical segmental comparison of end-systolic principal $\left(E_{\max }\right)$ and anatomical $\left(E_{r r}\right)$ strain estimations with five methods as compared to the ground truth. When considering the results in the pathological segment AS in all two subfigures of Fig. 7, the proposed methods exhibit good behavior, generally closely approaching the reference: IQR, median and mean values were similar to the ground truth with a slight under-estimation for $E_{\max }$ and $E_{r r}$.

Table 3 shows also the principal and anatomical strain error results of the compared methods. The average total strain errors of Sun's, Gao's, LK, Wang's, and MA methods were, respectively, $188 \%, 24 \%, 138 \%, 46 \%$, and $122 \%$ higher than the error of the proposed method when comparing the total error of the four types of strains. Similarly, Table 4 shows the principal and anatomical strain error results of the compared methods. The average error over the four strain measurements obtained by the Sun's, Gao's, LK, Wang's, and MA methods were, respectively, $92 \%, 26 \%, 57 \%, 29 \%$, and $38 \%$ higher than the error of the proposed method. Therefore, the proposed method has a better performance than other methods generally. From this simulation-based study, the good behavior of the proposed estimator on both C-MRI and T-MRI sequences can be observed, with results close to specialized methods in T-MRI.

\begin{tabular}{lcccc}
\hline & $E_{\max }$ & $E_{r r}$ & $E_{\min }$ & $E_{c c}$ \\
\hline Proposed & $\mathbf{0 . 0 4 8} \pm \mathbf{0 . 0 3 5}$ & $\mathbf{0 . 0 5 3} \pm \mathbf{0 . 0 4 0}$ & $\mathbf{0 . 0 2 9} \pm \mathbf{0 . 0 2 6}$ & $\mathbf{0 . 0 2 4} \pm \mathbf{0 . 0 2 8}$ \\
Sun & $0.116 \pm 0.079$ & $0.159 \pm 0.081$ & $0.106 \pm 0.070$ & $0.061 \pm 0.049$ \\
Gao & $0.061 \pm 0.040$ & $0.054 \pm 0.035$ & $0.040 \pm 0.029$ & $0.036 \pm 0.032$ \\
LK & $0.108 \pm 0.088$ & $0.118 \pm 0.081$ & $0.071 \pm 0.060$ & $0.070 \pm 0.072$ \\
Wang & $0.070 \pm 0.046$ & $0.068 \pm 0.036$ & $0.053 \pm 0.045$ & $0.034 \pm 0.036$ \\
MA & $0.100 \pm 0.061$ & $0.107 \pm 0.050$ & $0.085 \pm 0.067$ & $0.050 \pm 0.045$ \\
\hline
\end{tabular}

Table 3: Average error $(\mu \pm \sigma)$ of principal $\left(E_{\max }\right.$ and $\left.E_{\text {min }}\right)$ and anatomical $\left(E_{r r}\right.$ and $\left.E_{c c}\right)$ strains at end-systole over the whole myocardium from the TAG sequence.

\subsection{Preliminary results obtained on clinical data}

The method has been assessed on 5 clinical cases, including one healthy case and four pathological cases. All the clin- 
CINE Lagrangian endpoint error

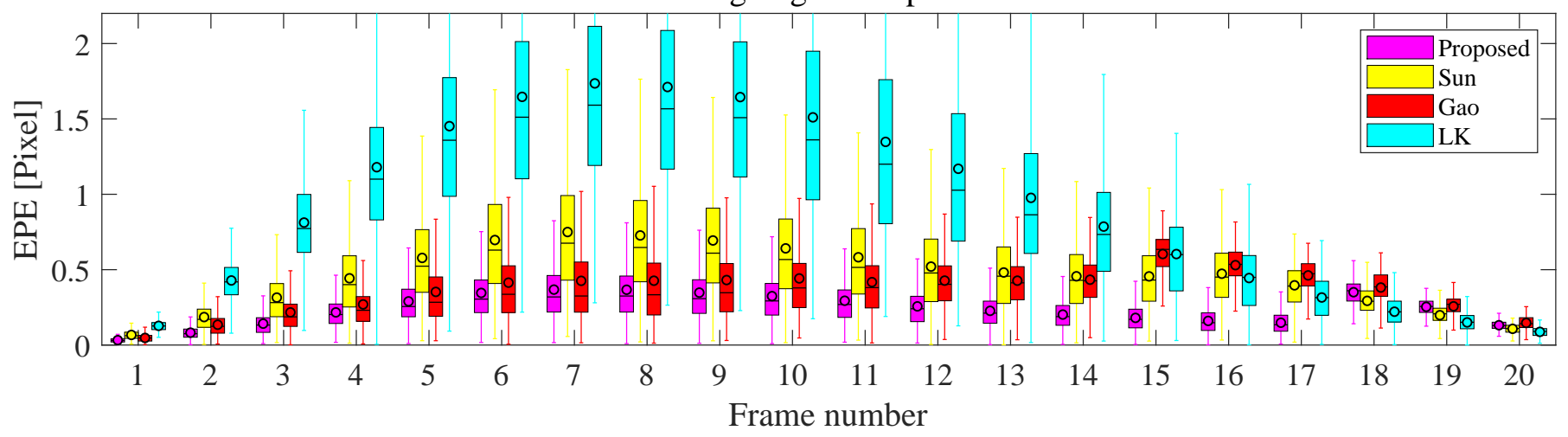

(a)

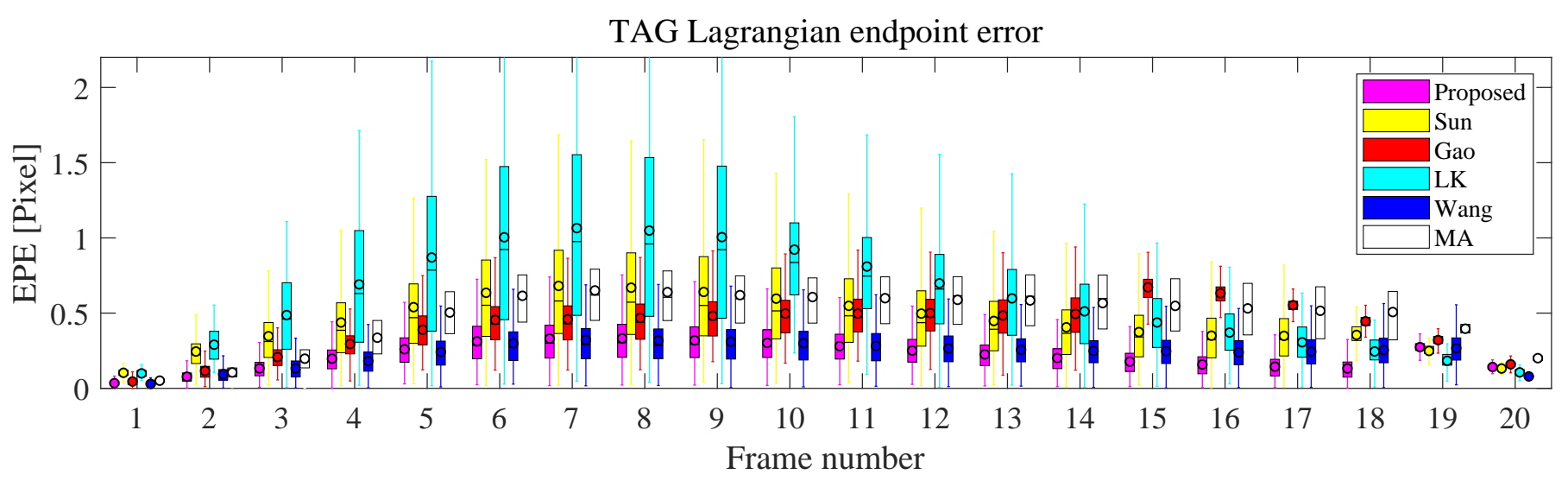

(b)

Figure 4: Box-and-whisker plot of Lagrangian EPE for the simulated sequences obtained with the proposed and other methods over the cardiac cycle. Each box represents the statistical distribution of EPE values at all time frames. The center bar of each box represents the median value, the circle indicates the mean value, and the box body extends from the 25th to the 75th percentile of EPE values. The lines extending vertically from the boxes indicate variability outside the upper and lower quartiles: (a) CINE; (b) TAG.

\begin{tabular}{lcccc}
\hline & $E_{\max }$ & $E_{r r}$ & $E_{\min }$ & $E_{c c}$ \\
\hline Proposed & $\mathbf{0 . 0 8 4} \pm \mathbf{0 . 0 6 9}$ & $\mathbf{0 . 0 8 0} \pm \mathbf{0 . 0 6 2}$ & $\mathbf{0 . 0 5 5} \pm \mathbf{0 . 0 5 2}$ & $\mathbf{0 . 0 5 0} \pm \mathbf{0 . 0 4 4}$ \\
Sun & $0.144 \pm 0.101$ & $0.178 \pm 0.097$ & $0.114 \pm 0.069$ & $0.081 \pm 0.075$ \\
Gao & $0.101 \pm 0.074$ & $0.085 \pm 0.063$ & $0.080 \pm 0.055$ & $0.073 \pm 0.055$ \\
LK & $0.139 \pm 0.111$ & $0.122 \pm 0.083$ & $0.086 \pm 0.072$ & $0.075 \pm 0.078$ \\
Wang & $0.106 \pm 0.075$ & $0.115 \pm 0.070$ & $0.069 \pm 0.052$ & $0.057 \pm 0.049$ \\
MA & $0.107 \pm 0.074$ & $0.104 \pm 0.062$ & $0.098 \pm 0.074$ & $0.063 \pm 0.055$ \\
\hline
\end{tabular}

Table 4: Average error $(\mu \pm \sigma)$ of principal $\left(E_{\max }\right.$ and $\left.E_{\min }\right)$ and anatomical $\left(E_{r r}\right.$ and $\left.E_{c c}\right)$ strains at end-systole over the whole myocardium from the TAG_FADING_NOISE sequence.

ical image data are from a study protocol approved by the Ethics Committee of our institution (IRB 123406519). All subjects gave written informed consent before inclusion (clinicaltrials.gov: NCT01208727). In this section, we present the results from one healthy volunteer and two patients. The results of two additional patients are presented in a complementary document (see Sec. 4.3.2).

The clinical short-axis C-MRI and T-MRI sequences were acquired at the same LV level on a Siemens Avento 1.5T using gradient echo (GRE) sequence. The parameters of the acquisition were for C-MRI sequences: $\mathrm{TR}=38.3 \mathrm{~ms}, \mathrm{TE}=1.74$ $m s$, flip angle $=70^{\circ}$, spatial resolution $=1.48 \mathrm{~mm}, 21$ frames, temporal resolution $=38.75 \mathrm{~ms}$; and for the T-MRI sequences: a $45^{\circ}$ SPAMM (spatial modulation of magnetization) tagging pattern, tag spacing $=6 \mathrm{~mm}, \mathrm{TR}=36.4 \mathrm{~ms}$, TE $=1.53 \mathrm{~ms}$, flip angle $=20^{\circ}$, spatial resolution $=1.09 \mathrm{~mm}, 21$ frames, temporal resolution $=36.4 \mathrm{~ms}$. Several recent methods used for comparison in the simulation cases in section 4.2 were applied on the clinical data, except the LK method that provides relatively less accurate simulation results. Since high accuracy of the Wang's and MA methods have been previously demonstrated in (Wang et al., 2015; Alessandrini et al., 2013) for T-MRI sequences, they were used as reference methods.

Figure 10(a) and Fig. 13(a) show the end-diastolic (first) frame of the C-MRI and T-MRI sequences in short axis orientation, respectively, at the medium level of the left ventricle of a healthy volunteer, which are the input data for the motion estimation algorithms. In addition, the motion estimation results obtained from two pathological cases are studied as well in the following. Masks and segment locations were validated by a clinical expert. 


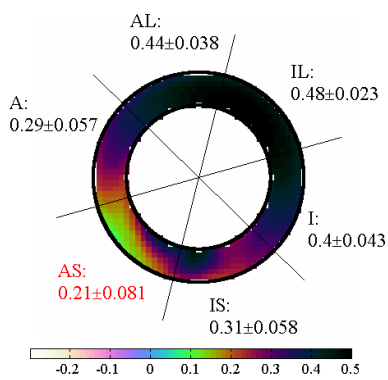

(a)

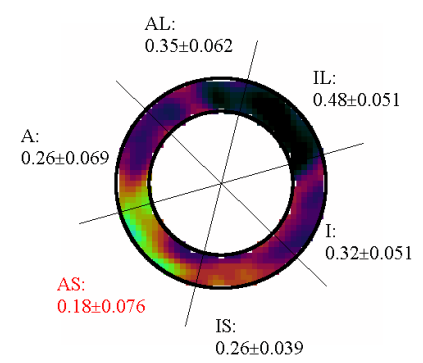

(b)

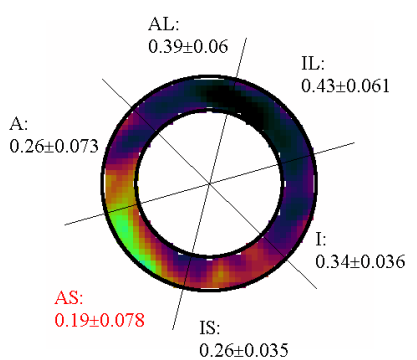

(c)

Figure 5: Maximum strain eigenvalue $E_{\max }$ maps sequence at end-systole. The red segment names indicate the pathological segments incorporated into the pathological simulation. (a) Ground truth; (b) proposed method with the CINE; (c) proposed method with the TAG;

\subsubsection{Results over the whole myocardium}

The proposed methods is evaluated in its ability to distinguish between normal and abnormal regions from both C-MRI and T-MRI, as compared to other approaches. Additionally, the ability of the proposed method to detect anomaly in C-MRI as compared to T-MRI is also considered.

Figures 8 and 9 show a general comparison of the average anatomical strains $\left(E_{r r}\right.$ and $\left.E_{c c}\right)$ for a healthy volunteer and four patients, with three methods applied on both C-MRI and T-MRI sequences. Globally, $E_{r r}$ and $E_{c c}$ absolute strain values are superior for the healthy case as compared to the pathological cases, even if it is less clear for patient \#3. It is observed that $E_{r r}$ values from C-MRI sequences are greater in magnitude than those of T-MRI sequences, except for patient \#4, but the three methods give the same trend. The contrary is observed for $E_{c c}$ in absolute values: C-MRI estimation is less than T-MRI estimation globally.

\subsubsection{Segmental results}

Healthy volunteer case. The myocardial strains (Figs. 10, 11, $13,14)$ were computed from the estimated Lagrangian motion field. The $E_{r r}$ maps obtained with the methods at end-systole are given in Fig. 10 and Fig. 13 from the C-MRI and T-MRI sequence, respectively. Smaller magnitude strains are observed from $E_{r r}$ in the anterior and inferior septal segments (AS and IS), which is generally the case in normal heart.

The proposed method presents results similar to the Gao's

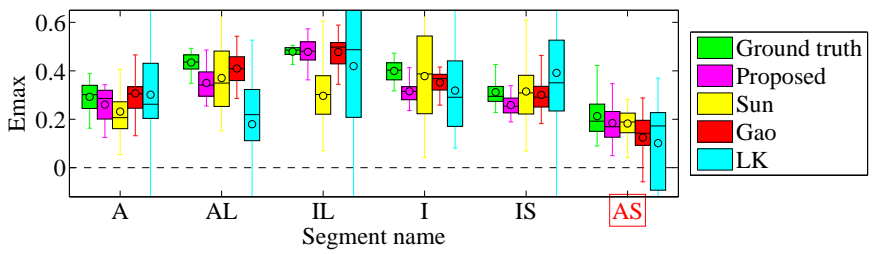

(a) Maximum strain eigenvalue $E_{\max }$

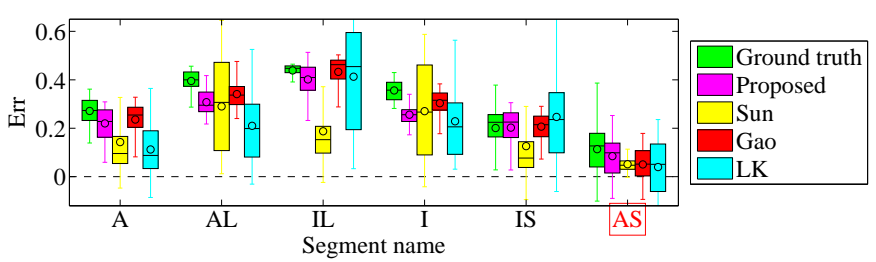

(b) Radial strain $E_{r r}$

Figure 6: Box-and-whisker plots of end-systolic principal $\left(E_{\max }\right)$ and anatomical $\left(E_{r r}\right)$ strains for the C-MRI CINE sequence. The segments highlighted with red text indicate the pathological segments.

method, and to the T-MRI specialized methods of Wang's and MA. C-MRI and T-MRI present the same relative tendency, but their magnitude was smaller in T-MRI (e.g. 0.42 in Fig. 10(c) for C-MRI vs. 0.2 in Fig. 13(c) for T-MRI sequence in the anterior segments). $E_{c c}$ maps are given in Fig. 11 and Fig. 14 for the C-MRI and T-MRI sequence, respectively. The interpretation and comparison of those maps is less easy. Considerable variation is observe when travelling along the myocardium for the proposed and Gao's method. $E_{c c}$ map for Sun is much more homogeneous, which probably comes from an over-smoothing by the median filtering in the motion estimation process (Sun et al., 2014).

Moreover, the statistical comparison for the anatomical $E_{r r}$ and $E_{c c}$ strains are represented in box-and-wiskers plots in Fig. 12 and Fig. 15. From Fig. 12, the proposed method shows more stable average value of strains than the other methods, which indicates the uniformity of motion behavior for a healthy myocardium. This uniformity can be also found in Fig. 15(b). As compared to the Wang's method, which was shown to produce very good estimation from T-MRI, the proposed method gave smaller $E_{r r}$ values in the segments A and AL.

Pathological case \#1. We studied the motion estimation results from a male patient (Patient \#1) with an inferior acute myocardial infarction (right coronary occlusion - reperfusion $\mathrm{H}+5$ ).

Fig. 16(a) shows the late gadolinium enhancement (LGE) short-axis image acquired at the medium level of the left ventricle. The hypersignal spans the IS, I, and IL segments, indicating the location of myocardial necrosis with a subendocardial no-reflow area in black.

Figures 16(b) and 18(a) show the first frame of the C-MRI and T-MRI pathological short axis (medium LV) sequences, respectively. Figures 16(d)-(f) show the distribution of the radial strain $E_{r r}$ extracted from the C-MRI and T-MRI sequences at end-systole. Significantly smaller $E_{r r}$ values are observed in the pathological inferior segments (IL, I, and IS) relatively to 


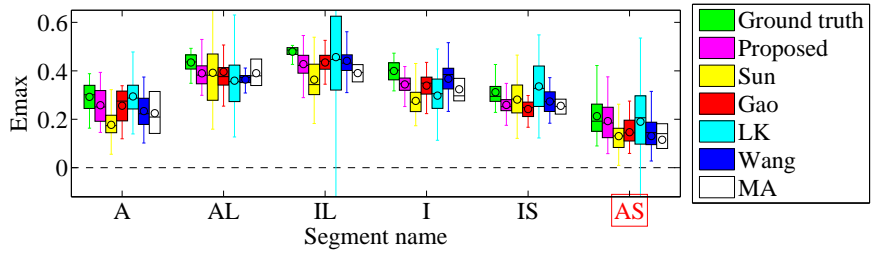

(a) Maximum strain eigenvalue $E_{\max }$

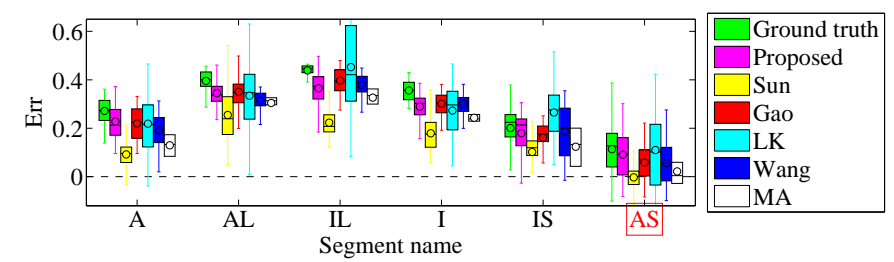

(b) Radial strain $E_{r r}$

Figure 7: Box-and-whisker plots of principal $\left(E_{\max }\right)$ and anatomical $\left(E_{r r}\right)$ strains for the T-MRI sequence TAG at end-systole. The segments highlighted with red text indicate the pathological segments.

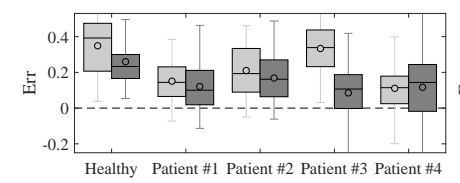

(a) Proposed

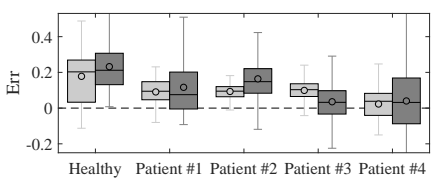

(b) Sun

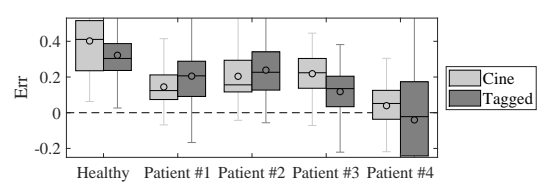

(c) Gao

Figure 8: Box-and-whisker plots of radial strain $E_{r r}$.

other segments $(0.056$ in the inferior segment vs 0.27 in the anterior segment of the C-MRI sequence in Fig. 16(d)) and to segments from the healthy case (0.056 in Fig. 16(d) vs 0.42 in Fig. 10(c) in the inferior segment). Smaller absolute values for $E_{r r}$ and $E_{c c}$ are clearly visible for pathological segments IL, I, and IS from box-and-whisker plots in Fig. 17.

In the corresponding T-MRI sequence, the same tendency can be observed. Figure 18(c)-(g) shows the distribution of the $E_{r r}$ maps extracted from the T-MRI sequence with the five methods at end-systole. In the pathological inferior segments (IL, I, and IS), the proposed method shows in Fig. 18(c) that the $E_{r r}$ value in the I segment is much lower than the $E_{r r}$ value of the normal A segment $(0.0059 \ll 0.21)$. Compared to the healthy T-MRI case in Fig. 13(c), the $E_{r r}$ value obtained in the I and A segments from the proposed method were 0.44 and 0.2 , respectively. In addition, reduced $E_{r r}$ values are observed in the identified pathological segments IS, I, and IL with most of the methods from Fig. 18(c), (d), (e), and (f). The lesion seems, in this pathological case and from T-MRIs, to impact at least partly the AS sector (Fig. 18(c-f)), even if it is difficult to discriminate between anomalous behavior or reduced motion in

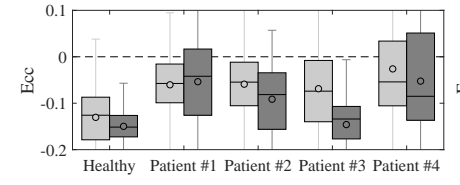

(a) Proposed

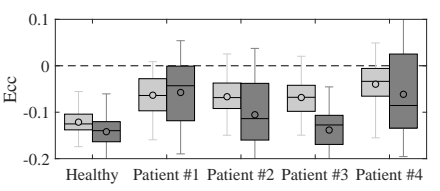

(b) Sun

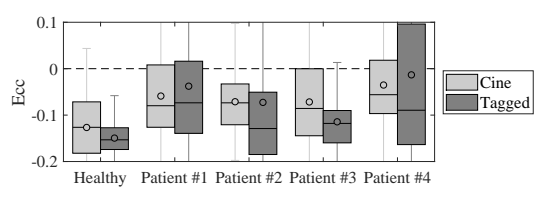

(c) Gao

Figure 9: Box-and-whisker plots of circumferential strain $E_{c c}$.

the septal area.

Differences between normal and abnormal regions are also clearly highlighted in the box-and-whisker plot in Fig. 19: magnitude of both $E_{r r}$ and $E_{c c}$ are closer to the zero line. Gao's method shows a much larger value extent in the IS segment.

Pathological case \#2. Results obtained for a second patient from C-MRI and T-MRI are given in Figs. 20 and 21, respectively. For this patient, segments IL and I has been identified as abnormal. The strains obtained with the proposed method can well distinguish the pathological segments IL and I from other segments. One can also observe that the proposed method outperforms the Sun's and Gao's methods when dealing with both the C-MRI and T-MRI sequences. With these cases, the proposed multiple gradient constrained method performs equally well on both cardiac C-MRI and T-MRI sequences as compared to state of the art methods.

Furthermore, the computing time of the proposed method is similar to the one of the Sun's method, while the overall EPE accuracy remains higher than Sun's, Gao's, LK, and MA methods, with a slightly lower accuracy than the Wang's method in T-MRI.

Generally, with Wang's and MA methods considered as a reference, the proposed method leads to similar results on C-MRI sequences than with corresponding T-MRI sequences and preserve the tendencies in the strain maps. Moreover, the clinical patients' results (patient \#3 and patient \#4) of strains from C-MRI and T-MRI were provided as complementary document. The warped T-MRI sequences and complementary document of all the patients can be found from https://www.creatis.insa-lyon.fr/ owncloud/index.php/s/rhCbkgRdQ50suBT. From the simulation study and the analysis of a first set of clinical cases, the proposed method shown to well adapt to both the C-MRI and TMRI sequences, and conducts to very similar motion estimation as compared to the reference methods.

\section{Conclusion}

We proposed a new myocardial motion estimation method that can be applied to both $2 D \mathrm{C}-\mathrm{MRI}$ and T-MRI sequences. 
It is based on the joint respect of local intensity distribution and intensity invariance during motion. The problem is formulated as an energy minimization where the local shape is approximated by four directional gradients. Minimization is achieved through a random walk scheme. The evaluation was first conducted on realistic simulated $2 D$ short-axis C-MRI and T-MRI sequences for which the true motion is known. The results show the very good accuracy obtained by the proposed method as compared to general-purpose motion estimators and more recent T-MRI specialized methods. Preliminary experiments conducted on clinical C-MRI and T-MRI data for healthy subject and patients show that the strain measurements obtained from the proposed motion estimator can efficiently differentiate healthy from anomalous myocardial regions in both modalities. Of course, the method's performance must now be assessed on a much larger data set to confirm those preliminary findings.

A clear potential of the proposed method is its good ability to quantify $2 D$ cardiac motion from conventional C-MRI, which is part of standard cardiac MRI protocols. Therefore, a huge amount of data can be retrospectively analyzed.

\section{Acknowledgments}

This study was conducted within the framework of the LABEX PRIMES (ANR-11-LABX-0063) and LABEX CELYA (ANR-10-LABX-0060) projects of the University of Lyon, within the "Investissements d'Avenir" (ANR-11-IDEX0007) program operated by the French National Research Agency (ANR).

\section{References}

Alessandrini, M., Basarab, A., Liebgott, H., Bernard, O., 2013. Myocardial motion estimation from medical images using the monogenic signal. IEEE Trans. Image Process. 22, 1084-1095.

Arts, T., Prinzen, F., Delhaas, T., Milles, J., Rossi, A., Clarysse, P., 2010. Mapping displacement and deformation of the heart with local sine-wave modeling. IEEE Trans. Med. Imag. 29, 1114-1123.

Bistoquet, A., Oshinski, J., Skrinjar, O., 2007. Left ventricular deformation recovery from cine MRI using an incompressible model. IEEE Trans. Med. Imag. 26, 1136-1153.

Boonsieng, P., Kondo, T., Kongprawechnon, W., 2014. A robust optical flow estimation technique using gradient orientations. ScienceAsia 40, 73-83.

Botella, G., Meyer-Baese, U., García, A., Rodríguez, M., 2012. Quantization analysis and enhancement of a VLSI gradient-based motion estimation architecture. Digit. Signal Process. 22, 1174-1187.

Bristow, H., Lucey, S., 2016. Dense Image Correspondences for Computer Vision. Springer International Publishing. chapter In Defense of GradientBased Alignment on Densely Sampled Sparse Features. pp. 135-152.

Brox, T., Bruhn, A., Papenberg, N., Weickert, J., 2004. High accuracy optical flow estimation based on a theory for warping, in: European Conference on Computer Vision, Springer. pp. 25-36.

Clarysse, P., Tafazzoli, J., Delachartre, P., Croisille, P., 2011. Simulation based evaluation of cardiac motion estimation methods in tagged-MR image sequences. J. Cardiovasc. Magn. Reson. 13, P360.

Delhay, B., Clarysse, P., Magnin, I., 2007. Locally adapted spatio-temporal deformation model for dense motion estimation in periodic cardiac image sequences, in: Functional Imaging and Modeling of the Heart, Salt Lake City, UT, USA. pp. 393-402.

Dosenbach, N.U., Koller, J.M., Earl, E.A., Miranda-Dominguez, O., Klein, R.L., Van, A.N., Snyder, A.Z., Nagel, B.J., Nigg, J.T., Nguyen, A.L., Wesevich, V., Greene, D.J., Fair, D.A., 2017. Real-time motion analytics during brain mri improve data quality and reduce costs. NeuroImage 161, 80 - 93 .

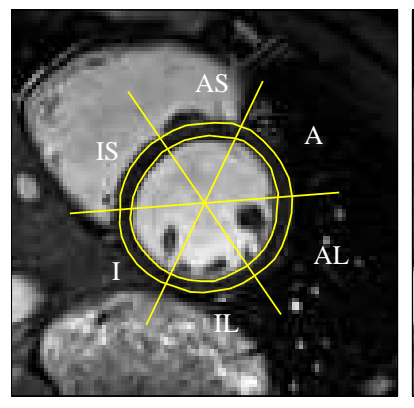

(a)

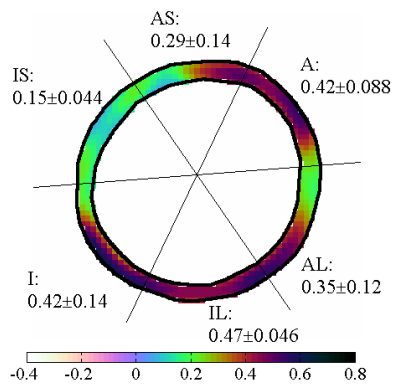

(c)

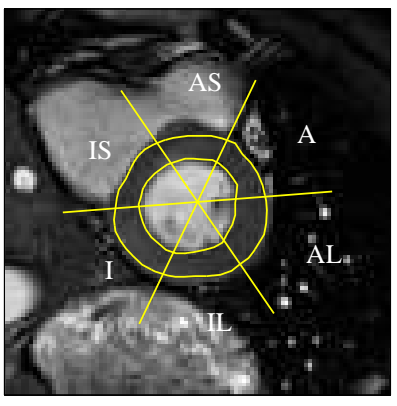

(b)

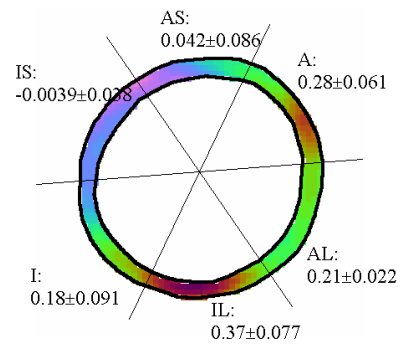

(d)

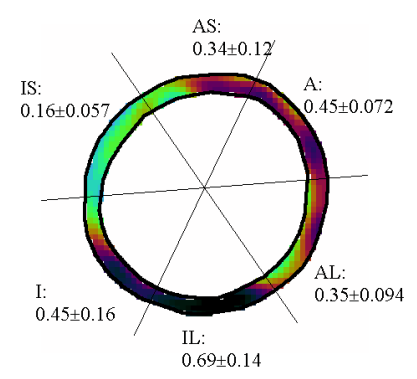

(e)

Figure 10: (a) End-diastolic (first) frame and (b) end-systolic frame of the CMRI sequence of a healthy volunteer with myocardial mask and segments delineated; (c)-(e) radial strain $E_{r r}$ maps at end-systole from the (c) proposed method; (d) Sun's method; (e) Gao's method.

Feng, L., Donnino, R., Babb, J., Axel, L., Kim, D., 2009. Numerical and in vivo validation of fast cine displacement-encoded with stimulated echoes (DENSE) MRI for quantification of regional cardiac function. Magn. Reson. Med. 62, 682-690

Fleet, D., Jepson, A., 1990. Computation of component image velocity from local phase information. Int. J. Comput. Vis. 5, 77-104.

Gao, B., Liu, W., Wang, L., Liu, Z., Croisille, P., Delachartre, P., Clarysse, P., 2016. Estimation of cardiac motion in cine-MRI sequences by correlation transform optical flow of monogenic features distance. Phys. Med. Biol. 61, 8640-8663.

Harris, C.M., 1989. The ethology of saccades: a non-cognitive model. Biological Cybernetics 60, 401-410.

Kawamoto, K., 2007. Optical flow-driven motion model with automatic variance adjustment for adaptive tracking, in: Asian Conference on Computer Vision, Springer Berlin Heidelberg. pp. 555-564.

Keller, Y., Averbuch, A., 2004. Fast motion estimation using bidirectional gradient methods. IEEE Trans. Imag. Process. 13, 1042-1054.

Küstner, T., Schwartz, M., Martirosian, P., Gatidis, S., Seith, F., Gilliam, C., Blu, T., Fayad, H., Visvikis, D., Schick, F., Yang, B., Schmidt, H., Schwen- 


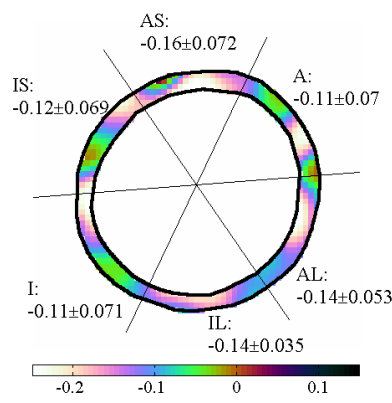

(a)

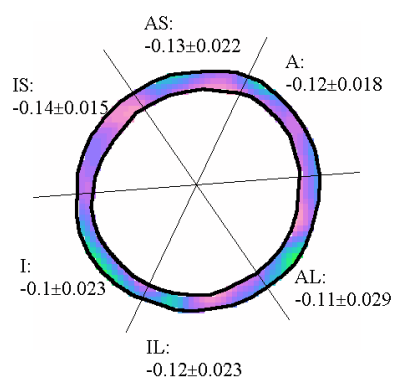

(b)

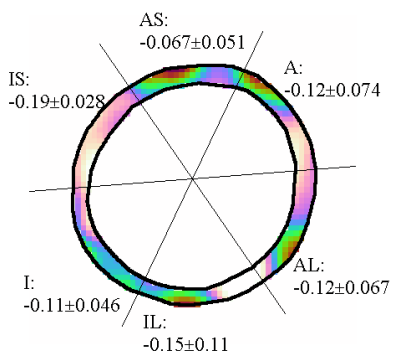

(c)

Figure 11: Circumferrential strain $E_{c c}$ maps at end-systole frame of the C-MRI sequence from a healthy volunteer from the (a) proposed method; (b) Sun's method; (c) Gao's method.

zer, N., 2017. Mr-based respiratory and cardiac motion correction for pet imaging. Med. Imag. Anal. 42, $129-144$.

Lucas, B.D., Kanade, T., 1981. An iterative image registration technique with an application to stereo vision, in: International Joint Conference on Artificial Intelligence, pp. 674-679.

Miao, S., Wang, Z., Pan, L., Butler, J., Moran, G., Liao, R., 2016. Scatter to volume registration for model-free respiratory motion estimation from dynamic mris. Comput. Med. Imag. Grap. 52, $72-81$.

Osman, N.F., Kerwin, W.S., Mcveigh, E.R., Prince, J.L., 1999. Cardiac motion tracking using CINE harmonic phase (HARP) magnetic resonance imaging. Magn. Reson. Med. 42, 1048-1060.

Oubel, E., De Craene, M., Hero, A.O., Pourmorteza, A., Huguet, M., Avegliano, G., Bijnens, B., Frangi, A.F., 2012. Cardiac motion estimation by joint alignment of tagged MRI sequences. Med. Image Anal. 16, 339-350.

Parages, F.M., Denney, T.S., Gupta, H., Lloyd, S.G., Dell'Italia, L.J., Brankov, J.G., 2017. Estimation of left ventricular motion from cardiac gated tagged mri using an image-matching deformable mesh model. IEEE Trans. Radiat. Plasma Med. Sci. 1, 147-157.

Prince, J., McVeigh, E., 1992. Motion estimation from tagged MR image sequences. IEEE Trans. Med. Imag. 11, 238-249.

Qian, Z., Liu, Q., Metaxas, D., Axel, L., 2011. Identifying regional cardiac abnormalities from myocardial strains using nontracking-based strain estimation and spatio-temporal tensor analysis. IEEE Trans. Med. Imag. 30, 2017-2029.

Revaud, J., Weinzaepfel, P., Harchaoui, Z., Schmid, C., 2015. EpicFlow: Edgepreserving interpolation of correspondences for optical flow, in: IEEE Conference on Computer Vision and Pattern Recognition, pp. 1164-1172.

Sun, D., Roth, S., Black, M., 2010. Secrets of optical flow estimation and their principles, in: IEEE Conference on Computer Vision and Pattern Recognition, pp. 2432-2439.

Sun, D., Roth, S., Black, M., 2014. A quantitative analysis of current practices in optical flow estimation and the principles behind them. Int. J. Comput. Vis. 106, 115-137.

Sundar, H., Litt, H., Shen, D., 2009. Estimating myocardial motion by 4D

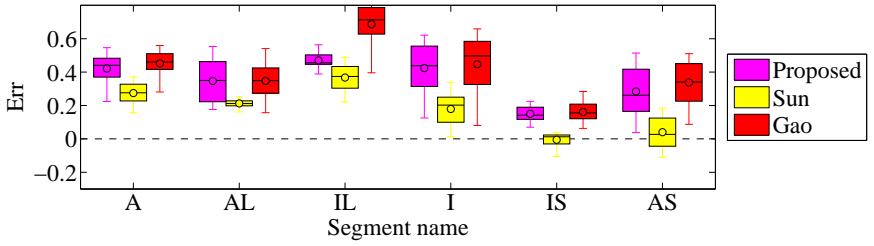

(a)

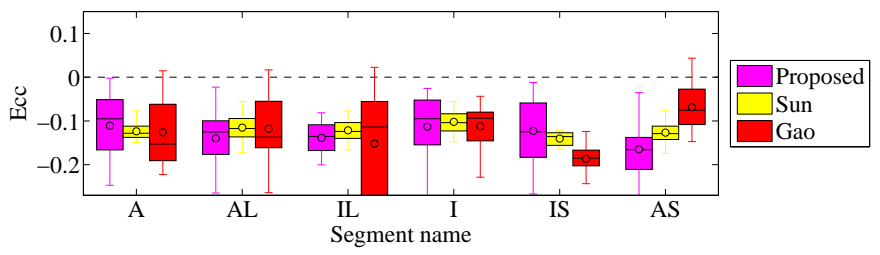

(b)

Figure 12: Box-and-whisker plot of C-MRI sequence for the Healthy case (a) $E_{r r}$; (b) $E_{c c}$.

image warping. Pattern Recognition 42, 2514-2526.

Tecelão, S.R., Zwanenburg, J.J., Kuijer, J.P., Marcus, J.T., 2006. Extended harmonic phase tracking of myocardial motion: Improved coverage of myocardium and its effect on strain results. J. Magn. Reson. Imaging 23, 682690.

Tolouee, A., Alirezaie, J., Babyn, P., 2018. Nonrigid motion compensation in compressed sensing reconstruction of cardiac cine MRI. Magn. Reson. Imaging 46, $114-120$.

Wang, H., Amini, A.A., 2012. Cardiac motion and deformation recovery from MRI: A review. IEEE Trans. Med. Imag. 31, 487-503.

Wang, L., Basarab, A., Girard, P., Croisille, P., Clarysse, P., Delachartre, P., 2015. Analytic signal phase-based myocardial motion estimation in tagged MRI sequences by a bilinear model and motion compensation. Med. Imag. Anal. 24, 149-162.

Xu, C., Pilla, J.J., Isaac, G., Gorman, J.H., Blom, A.S., Gorman, R.C., Ling, Z., Dougherty, L., 2010. Deformation analysis of 3D tagged cardiac images using an optical flow method. J. Cardiovasc. Magn. Reson. 12, 1-14.

Zang, D., Wietzke, L., Schmaltz, C., Sommer, G., 2007. Dense optical flow estimation from the monogenic curvature tensor, in: Scale Space and Variational Methods in Computer Vision. Springer Berlin Heidelberg. volume 4485 of Lecture Notes in Comput. Sci., pp. 239-250.

Zhou, Y., Giffard-Roisin, S., De Craene, M., Camarasu-Pop, S., D’ Hooge, J., Alessandrini, M., Friboulet, D., Sermesant, M., Bernard, O., 2018. A framework for the generation of realistic synthetic cardiac ultrasound and magnetic resonance imaging sequences from the same virtual patients. IEEE Trans. Med. Imag. 37, 741-754.

Zimmer, H., Bruhn, A., Weickert, J., 2011. Optic flow in harmony. Int. J. Comput. Vis. 93, 368-388. 


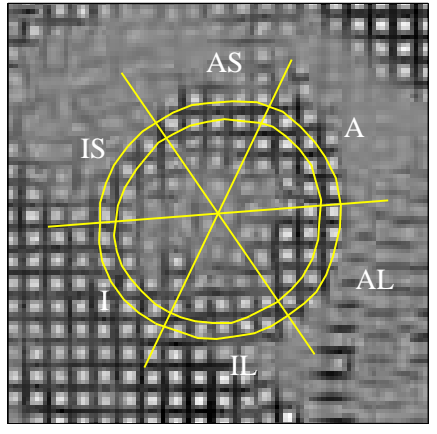

(a)

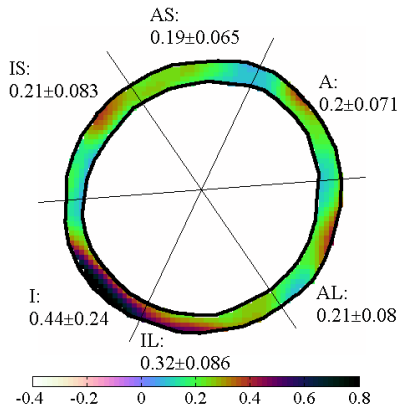

(c)

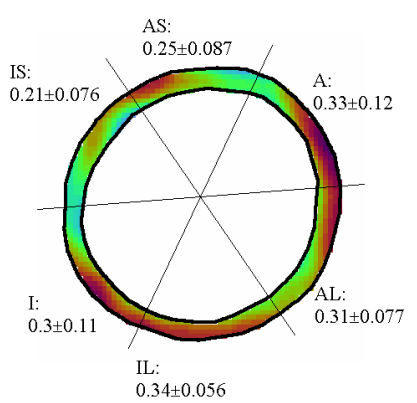

(e)

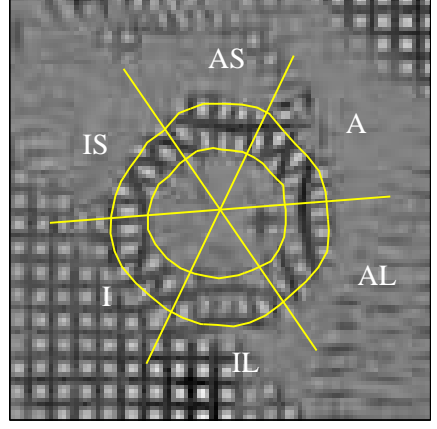

(b)

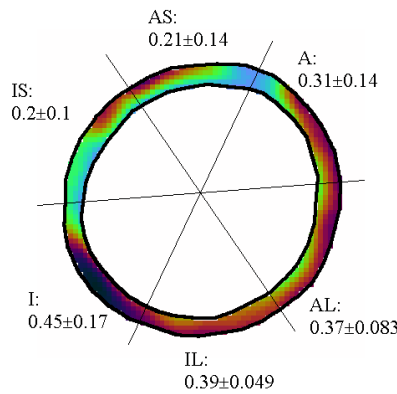

(d)

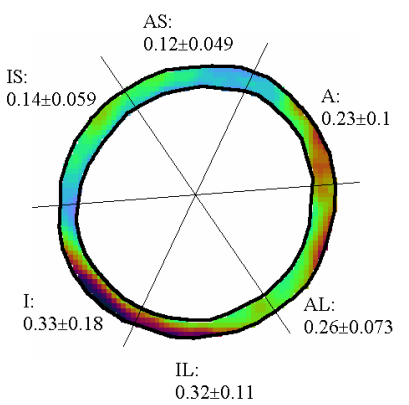

(f)

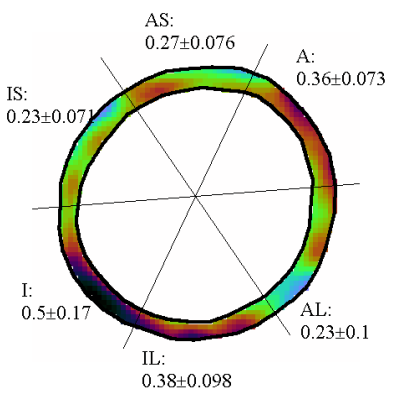

(g)

Figure 13: (a) End-diastolic (first) frame and (b) end-systolic frame of the TMRI sequence for the healthy volunteer with myocardial mask and segments delineated; (c)-(g) radial strain $E_{r r}$ maps from the (c) proposed method; (d) Wang's method; (e) MA method; (f) Sun's method; (g) Gao's method.

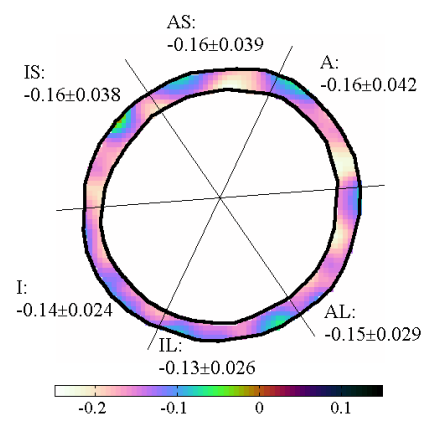

(a)

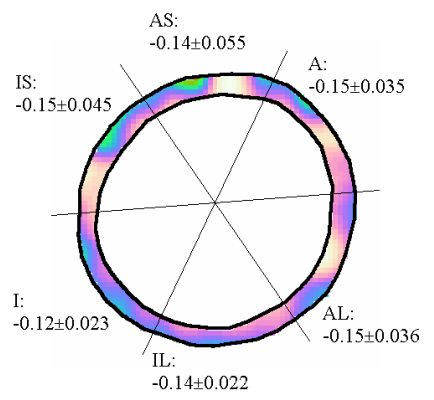

(c)

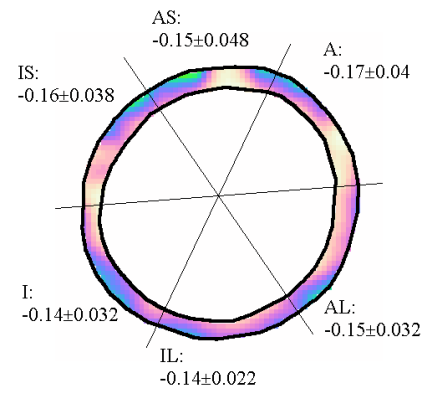

(b)

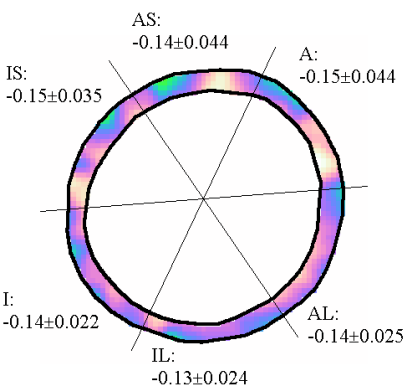

(d)

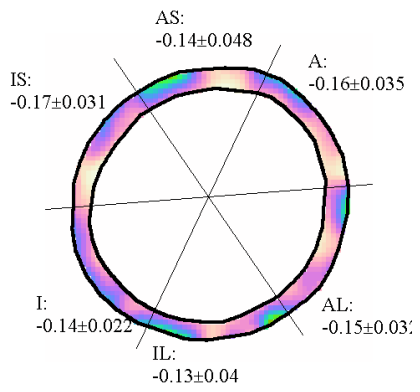

(e)

Figure 14: Circumferrential strain $E_{c c}$ maps at end-systole frame of the TMRI sequence from a healthy volunteer from the the (a) proposed method; (b) Wang's method; (c) MA method; (d) Sun's method; (e) Gao's method. 


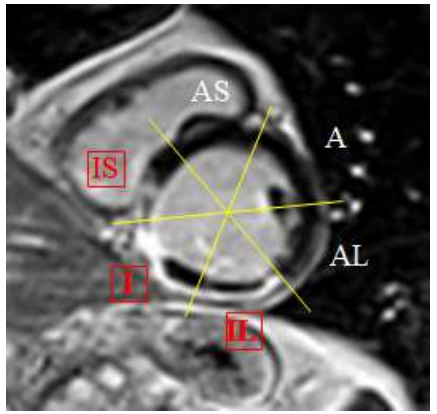

(a)

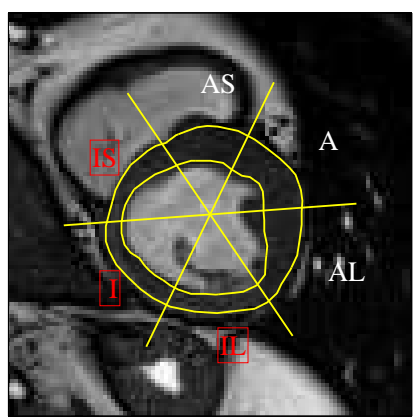

(c)

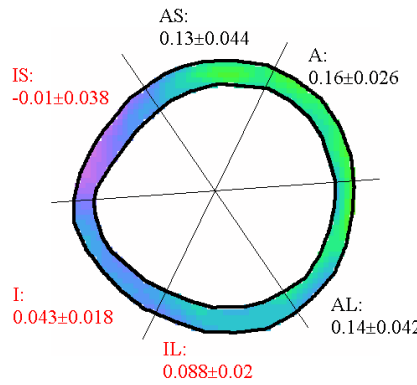

(e)

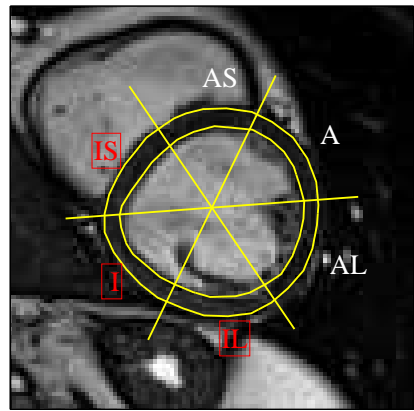

(b)

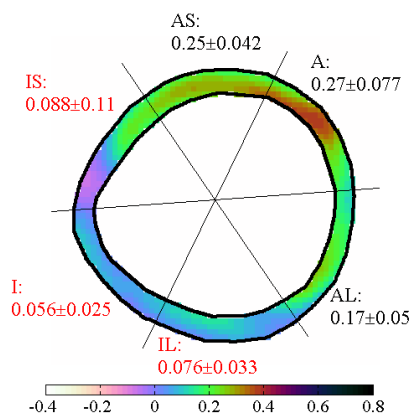

(d)

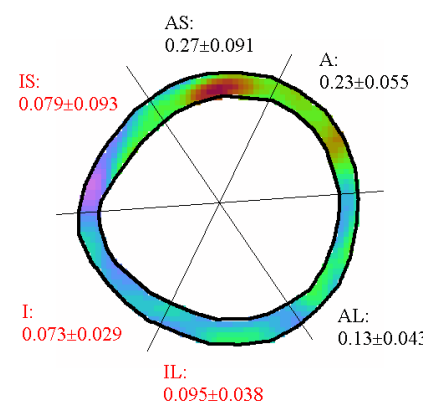

(f)

Figure 16: Patient \#1: (a) LGE MR short axis image showing the necrosed region in hypersignal spreading within the IS, I, and IL myocardial segments of the pathological case; (b) end-diastolic (first) frame, and (c) end-systolic frame of the pathological C-MRI sequence with myocardial mask and segments delineated (pathological ones in red and others in white); (d)-(f) radial strain $E_{r r}$ maps at end-systole obtained with the: (d) proposed method; (e) Sun's method; (f) Gao's method. Pathological segments are indicated with red text. 


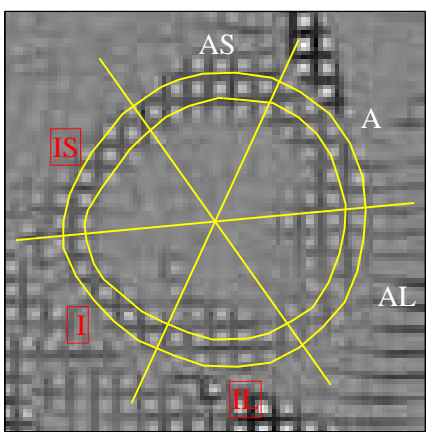

(a)

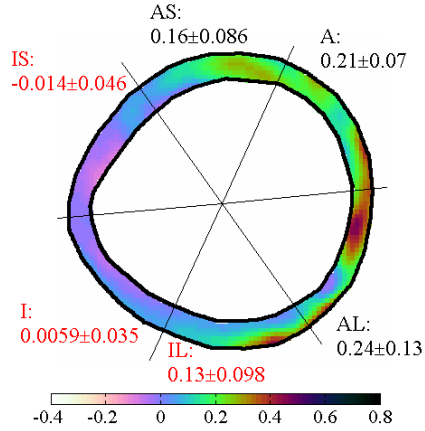

(c)

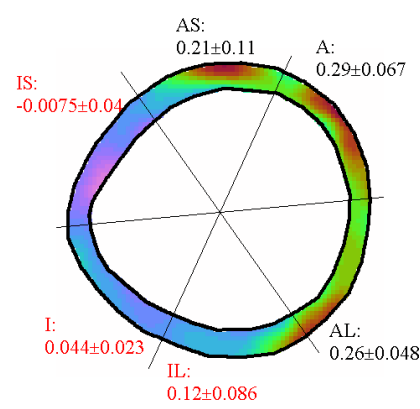

(e)

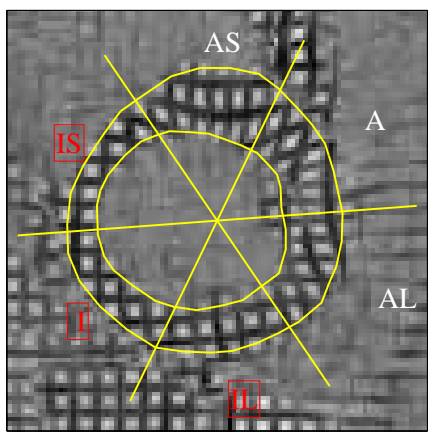

(b)

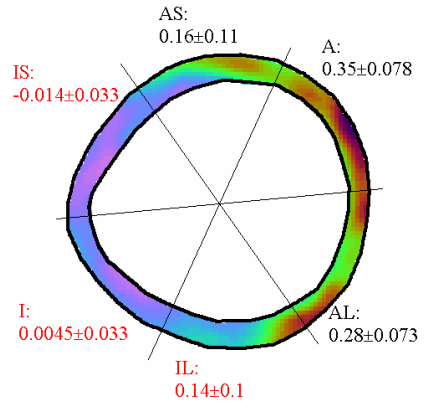

(d)

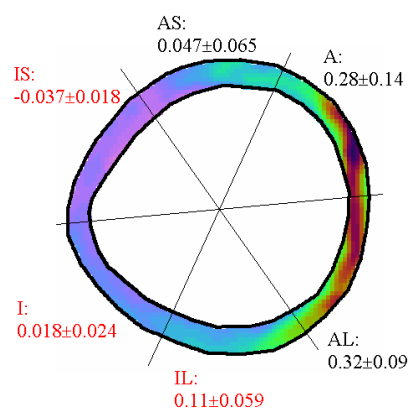

(f)

Figure 17: Box-and-whisker plot of C-MRI sequence for Patient \#1: (a) $E_{r r}$; (b) $E_{c c}$.

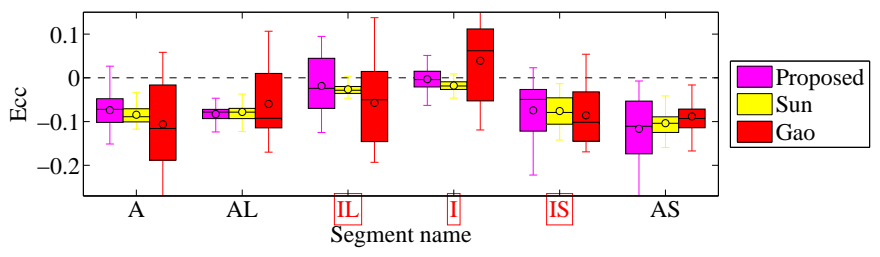

(b)

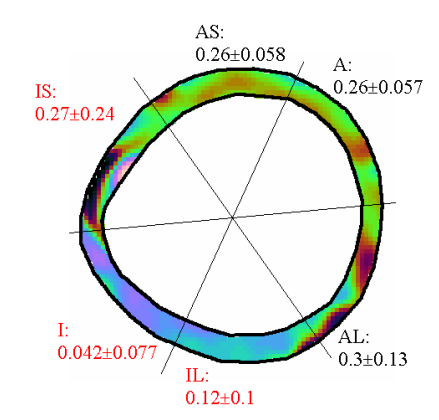

(g)

Figure 18: Patient \#1: (a) end-diastolic (first) frame, and (b) end-systolic frame of the pathological T-MRI sequence with myocardial mask and segments (pathological segments in red) indicated; (c)-(g) radial strain $E_{r r}$ maps from the methods with the red segment names indicating the pathological segments: (c) proposed method; (d) Wang's method; (e) MA method; (f) Sun's method; (g) Gao's method. 


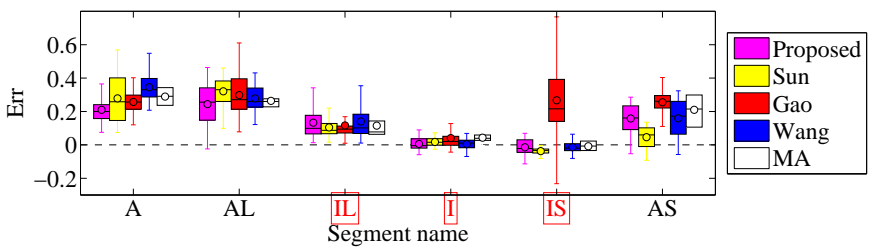

(a)

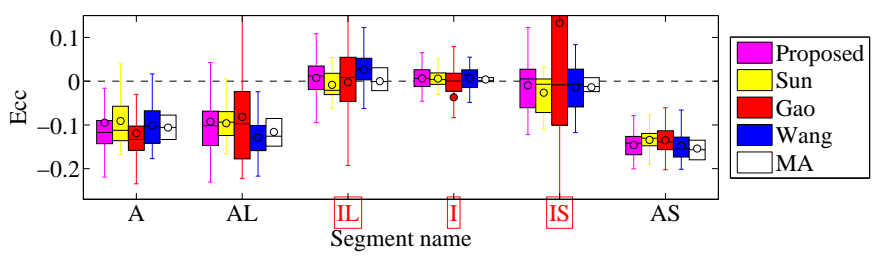

(b)

Figure 19: Box-and-whisker plot of T-MRI sequence for Patient \#1:(a) $E_{r r}$; (b) $E_{c c}$.

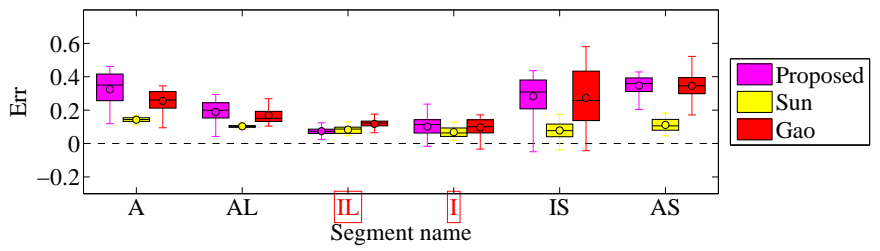

(a)

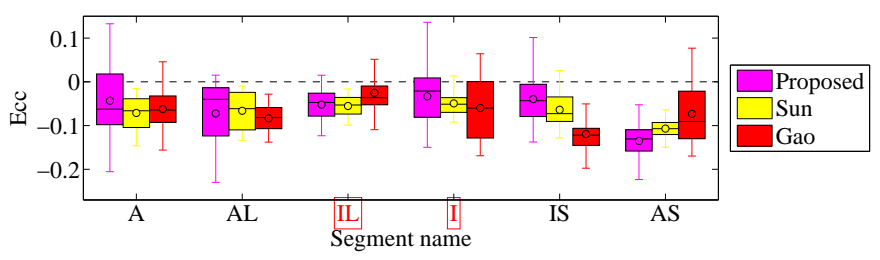

(b)

Figure 20: Box-and-whisker plot of C-MRI sequence for Patient \#2: (a) $E_{r r}$; (b) $E_{c c}$

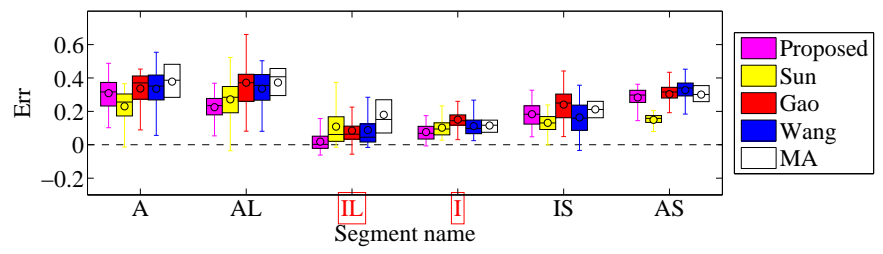

(a)

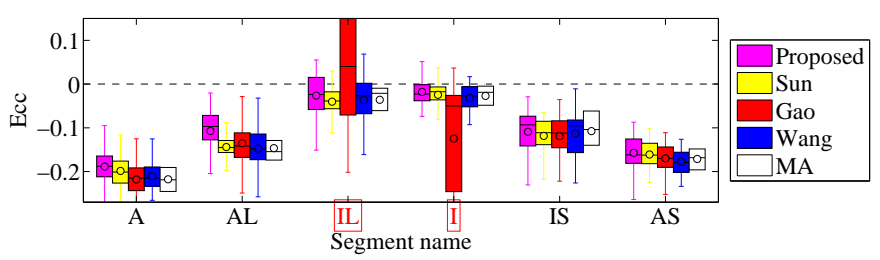

(b)

Figure 21: Box-and-whisker plotof T-MRI sequence for Patient \#2: (a) $E_{r r}$; (b) $E_{c c}$. 